\title{
El cine-foro virtual en la formación docente: experiencia didáctica con estudiantes de Educación Primaria en la Universidad de Costa Rica, Sede Rodrigo Facio
}

\author{
Virtual Film Forum in teacher training: didactic experience with Primary Education \\ students at the University of Costa Rica's Rodrigo Facio Headquarters \\ O cine-fórum virtual na formação de professores: uma experiência didática com alunos \\ do Ensino Fundamental na Universidade da Costa Rica, Campus Rodrigo Facio
}

\author{
Esteban Francisco Ibarra-Vargas \\ Universidad de Costa Rica \\ San José, Costa Rica \\ esteban.ibarra@ucr.ac.cr \\ (D) https://orcid.org/0000-0001-5915-5101
}

Recibido - Received - Recebido: 06 / 10 / 2020 Corregido - Revised - Revisado: 12 / 03 / 2021 Aceptado - Accepted - Aprovado: 12 / 04 / 2021

\author{
DOI: https://doi.org/10.22458/ie.v23i34.3242 \\ URL: https://revistas.uned.ac.cr/index.php/innovaciones/article/view/3242
}

\begin{abstract}
Resumen: La sistematización de este trabajo presenta los resultados de la valoración efectuada por el estudiantado participante en la experiencia didáctica Cine-foro virtual, llevada a cabo en el curso Educación Rural y Escuela Unidocente, en la Universidad de Costa Rica. La ejecución correspondió con la necesidad de sustituir una gira educativa de inmersión presencial en el contexto rural unidocente, debido a la pandemia ocasionada por el virus responsable de la COVID-19. Para efectuar esta labor, se parte del enfoque socio-crítico y la pedagogía crítica, se propone abordar los conceptos de estrategia didáctica y portafolio de evidencias, para argumentar el uso del Cine-foro virtual; además se describen aspectos de la escuela unidocente y la persona docente. En el marco de una investigación cualitativa que estudia la perspectiva del estudiantado participante en la experiencia, se destacan como principales resultados que: a pesar de la no inmersión en el contexto de desempeño deseado, la experiencia virtual fue exitosa para el alcance del objetivo propuesto; facilitó, por medio del diálogo social participativo, la construcción de una comunidad de aprendizaje donde fue posible compartir conocimientos, experiencias e inquietudes respecto a la realidad educativa rural unidocente; que la secuencia de actividades innova en la práctica docente universitaria y esta se puede adaptar a diversos cursos y contextos de aprendizaje en el área educativa.
\end{abstract}

Palabras Clave: Formación profesional, Educación rural, Docente de escuela primaria, Estrategias educativas, Aprendizaje en línea, COVID-19

\begin{abstract}
Summary: The systematization of this work presents the results of the evaluation carried out by the participating students in the didactic experience Virtual Film Forum, carried out in the Rural Education course and the singleteacher school at the University of Costa Rica. It all happened when faced with the need to replace a face-toface immersion educational tour in the rural single-teacher school context due to the pandemics caused by the virus responsible for COVID-19. To carry out this work, we adopted a socio-critical approach and benefited from critical pedagogy. The concepts of didactic strategy and portfolio of evidence we readdressed to discuss the advantage of the Virtual Film Forum. In addition, aspects of the single-teacher school and the teacher are described. Within the framework of qualitative research that studies the perspective of the student body participating in the experience, the main results showed that: despite the students not being immersed in the desired performance context, the virtual experience was successful in attaining the outlined objective; it also facilitated, through participatory social dialogue, the construction of a learning community where it was possible to share knowledge, experiences, and concerns regarding the reality of the rural single-teacher school. That sequence of activities innovates in University teaching practice and can be adapted to various courses and learning contexts in the educational domain.
\end{abstract}

Keywords: vocational training, rural education, primary school teacher, educational strategies, online learning, COVID-19 
Resumo: A sistematização deste trabalho apresenta os resultados da avaliação feita pelos alunos que participaram da experiência didática Cine-foro virtual, realizada no curso Educação Rural e na escola de um professor, na Universidade da Costa Rica. A execução correspondeu à necessidade de substituir um tour educativo de imersão presencial no contexto rural de um professor, devido à pandemia causada pelo vírus responsável pela COVID-19. Para realizar este trabalho, partimos da abordagem sócio crítica e pedagogia crítica, propomos abordar os conceitos de estratégia didática e portfólio de evidências, para argumentar o uso do Cine-fórum virtual; além disso, descrevemos aspectos da escola de um professor e do professor. No âmbito de uma pesquisa qualitativa que estuda a perspectiva dos estudantes participantes da experiência, os principais resultados são: apesar da falta de imersão no contexto de desempenho desejado, a experiência virtual foi bem sucedida na realização do objetivo proposto; facilitou, através do diálogo social participativo, a construção de uma comunidade de aprendizagem onde foi possível compartilhar conhecimentos, experiências e preocupações sobre a realidade da educação rural de um professor; que a sequência de atividades inova na prática do ensino universitário e pode ser adaptada a vários cursos e contextos de aprendizagem na área educacional.

Palavras chave:formação profissional, educação rural, professor do ensino fundamental, estratégias educacionais, aprendizagem on-line, COVID-19

\section{INTRODUCCIÓN}

El propósito del documento es compartir con la población docente y académica, en general, la sistematización de la experiencia de una práctica de aula realizada durante el I ciclo de 2020, en el curso Educación Rural y Escuela Unidocente, impartido por la Escuela de Formación Docente al estudiantado que se forma en la carrera de Licenciatura en Educación Primaria, en la Facultad de Educación de la Universidad de Costa Rica (UCR), Sede Rodrigo Facio.

Ante la situación ocasionada por el virus responsable de la COVID-19, la Rectoría de la UCR, según la resolución (R-95-2020, 2020), resuelve suspender todas las actividades presenciales como una medida para la atención, a nivel institucional, de la alerta sanitaria; por ello, la experiencia se diseñó y ejecutó en una modalidad virtual-asincrónica.

El problema radica en que, ante la virtualización de las actividades del curso, se imposibilitó que el estudiantado asistiera a una gira educativa programada en la modalidad presencial, como una estrategia de inmersión directa en el contexto rural-unidocente, la cual posibilitaría la adquisición de conocimientos y aprendizajes vinculados con la unidocencia.

Dicha gira fue concebida como una actividad que se sustenta en el enfoque socio-crítico y la pedagogía crítica, fundamentos epistemológicos en el quehacer en la carrera de Educación Primaria. Respecto al enfoque socio-crítico, la Sección de Educación Primaria (2014), afirma que este promueve la "construcción del conocimiento a partir de la interacción, mediante experiencias de vida y las características socioculturales del entorno" (p. 32).

Por otro lado, la Sección de Educación Primaria, también alude a la pedagogía crítica como un enfoque que "permite abordar y afrontar el hecho educativo desde la investigación, la reflexión y la toma de conciencia, desde una nueva óptica de visualizar la realidad circundante" (Sección de Educación Primaria, 2014, p. 35). Por lo tanto, se enfatiza en la importancia de que las actividades de formación docente, como, por ejemplo, las giras educativas, promuevan la relación teoría-práctica y acción-reflexión.

En este contexto, se aborda la interrogante: ¿cómo lograr que el estudiantado del curso Educación Rural y Escuela Unidocente, por medio de una actividad virtual asincrónica, comprenda la relación teóricopráctica y de acción-reflexión sobre la realidad de las escuelas unidocentes y el papel de la persona docente?

Para atender la situación se promovió la planificación de la estrategia Cine-foro virtual, la cual se hace acompañar de varios recursos didácticos que facilitan aprendizajes direccionados desde ese contacto 
entre la teoría y la posible práctica pedagógica unidocente, así como la acción-reflexión sobre la realidad de las escuelas unidocentes y el papel del profesorado.

Para contextualizar la experiencia, el Cine-foro virtual se asume como una estrategia didáctica, la cual, como señala Tobón (2005), citado en DEDUN (2020), se trata de "un conjunto de procedimientos, apoyados en técnicas, que tiene por objeto llevar a buen término la acción didáctica, es decir, alcanzar los objetivos de aprendizaje propuestos, con clara y explícita intencionalidad pedagógica" (p. 4). Para su ejecución se siguen instrucciones que guían los diferentes momentos de su aplicación, se circunscriben elementos de la evaluación diagnóstica, formativa y sumativa, además de proyectarse hacia los resultados que se desean obtener con la participación de la población estudiantil.

La experiencia incorpora la construcción grupal de un portafolio de evidencias en formato digital, el cual se concibe como una excelente herramienta de aprendizaje que complementa la secuencia didáctica y fomenta los procesos metacognitivos y el uso de estrategias mnemotécnicas en el estudiantado; a la vez que promueve diversas formas de organización de la información relevante para su propio aprendizaje.

Barberá (2005) se refiere a la estrategia del portafolio, indicando que "[...] es una colección organizada de trabajos y documentos previamente seleccionados por el alumno y que reflejan su proceso y su rendimiento en relación con unos objetivos de aprendizaje y unos criterios de evaluación preestablecidos" (p. 499). Por lo tanto, este recurso que elabora el estudiantado se convierte en un material de consulta al que se puede acceder durante la práctica pedagógica profesional futura.

Ahora bien, el uso del foro virtual se promueve en virtud de su flexibilidad en tiempo y espacio; pues la población estudiantil puede realizar aportes en diferentes líneas de discusión y momentos del proceso, además de que brinda la posibilidad de realizarse de forma asincrónica. Acorde con el tema, Arango (2003) considera que

En el ámbito educativo, el ejercicio asincrónico propio de los foros virtuales permite a los estudiantes articular sus ideas y opiniones desde distintas fuentes de discusión, promoviendo el aprendizaje a través de varias formas de interacción distribuidas en espacios y tiempos diferentes. (p. 3).

Si se toma en cuenta lo anterior, se considera que el foro debe acompañarse por otro tipo de recursos que conduzcan hacia la activación de aprendizajes previos y posteriormente, procesos de reflexión y análisis sobre los objetivos por alcanzar. Por esta razón, la experiencia se acompaña de un proceso de lectura y la observación de un documental, recursos que facilitan el acercamiento al contenido curricular, para fomentar, por medio del trabajo cooperativo, la discusión y el diálogo entre el estudiantado.

Desde la perspectiva de la persona autora de este documento y tomando en cuenta los argumentos que anteceden, se conceptualiza el Cine-foro virtual como una estrategia que se compone por actividades virtuales asincrónicas que se desarrollan en el marco de la observación de un material audiovisual, esto permite al estudiantado tomar una postura crítica sobre el objetivo y el contenido curricular por tratar, y en una dinámica de interacción social, analizar y reflexionar en torno a la relación teoría-práctica que se concretiza por medio de las vivencias y experiencias relacionadas con el objeto de estudio.

Por consiguiente, el Cine-foro virtual se destaca como un medio que posibilita el diálogo entre participantes, un espacio que no puede darse de manera presencial en el escenario universitario actual, pero que es necesario realizar porque el aprendizaje es inminentemente social.

Piedra (2010), en relación con este factor social de la educación y particularmente sobre los procesos de formación universitaria, indica que, "deberían estar encaminados a crear comunidades de conocimiento en donde el docente no solamente facilite conocimientos puntuales sino también habilidades para el trabajo grupal y cooperativo." (p. 318). 
La experiencia, por lo tanto, motiva una estrategia de interacción estudiantil que reconoce que los aprendizajes son efectivamente sociales y posibles de desarrollar por medio de una educación a distancia; mientras se crean, a pequeña escala, comunidades de conocimiento que van más allá de la adquisición de supuestos en un escenario de presencialidad física.

En el desarrollo de la experiencia se aborda el tema de las escuelas unidocentes. Para Cartín (2018), estas son

[...] instituciones de I-II Ciclos que albergan una población escolar de 30 o menos alumnos de diferentes niveles, los cuales son atendidos por un solo docente. Estas escuelas se encuentran en todo el territorio nacional y en su mayoría se caracterizan por estar ubicadas en las zonas rurales y dispersas del país. (p. 21).

A pesar de que actualmente se refleja una importante disminución en el porcentaje de escuelas unidocentes de Costa Rica; pues para el 2007 estas escuelas representaban el 49,63\% de la totalidad de centros educativos del país (Chaves y García, 2013) y para el 2017, alcanzaron un 39,5\% (Programa Estado de la Nación, 2017), esto representa una realidad educativa que el estudiantado en formación docente debe conocer.

A las escuelas unidocentes también se les conoce como escuelas multigrado (Chaves y García, 2013). Bustos (2011), en relación con la denominación de escuela multigrado, menciona que "La podemos definir como un tipo de agrupamiento escolar en el que el alumnado de diferentes grados comparte las condiciones del ambiente de aprendizaje." (p. 81). Es así como estas escuelas se caracterizan por un nivel bajo de matrícula y ser atendidas por una persona docente quien asume responsabilidades educativas con el estudiantado de los seis niveles educativos del I y II Ciclos de la Educación General Básica, así como ejercer la gestión administrativa en estrecho vínculo con la comunidad, donde desarrolla experiencias de liderazgo. Tal como señalan Alvarado y Maya (2009),

[...] el maestro o maestra de escuelas unidocentes, asume un papel muy diferente al de otras instituciones, ya que debe planificar el trabajo diario de tal forma que pueda estimular, orientar y mediar los aprendizajes de una manera no directiva, ya que no puede atender directamente a todos los niveles a la vez. (p. 15).

Como la educación es intencionada y en su accionar repercute una serie de aspectos pedagógicos que conllevan la enseñanza y la adquisición de aprendizajes, se asume que la pedagogía, como disciplina que estudia la educación, utiliza como materia prima los hechos y procesos pedagógicos (Alvarado y Maya, 2009), lo cual posibilita la construcción de teoría educativa y la orientación didáctica para el desarrollo de la acción pedagógica de la persona unidocente, aspectos fundamentales en el proceso de aprendizaje del estudiantado que participa en esta experiencia didáctica.

Por consiguiente, en el proceso de análisis y reflexión que se pretende generar, resulta fundamental considerar, de forma transversal, los pilares que orientan y fundamentan la práctica educativa multigradounidocente, los cuales abordan las personas autoras antes mencionadas:

a) epistemológico: relacionado con la orientación del proceso educativo y el ejercicio de toma de decisiones respecto a qué enseñar.

b) pedagógico: el cual refiere al conocimiento y a la forma en que se aplican las estrategias didácticas en el contexto unidocente.

c) psicosocial: relativo a la indagación sobre el contexto y las características psicológicas y sociales de la comunidad y del estudiantado. 
d) psicopedagógico: relacionado con el ejercicio de comprensión y adaptación de las condiciones de aprendizaje al estudiantado, tomando en cuenta las características de cada sujeto y centro educativo, así como la diversidad cultural.

e) axiológico: el cual se vislumbra en la enseñanza y el aprendizaje de valores, a través de las situaciones cotidianas en las que participa la persona docente y el estudiantado.

En síntesis, la tarea unidocente no es sencilla. Se debe ser una persona creativa, crítica, reflexiva, capaz de contextualizar el currículo a la realidad de la comunidad, debe organizar y planificar sus clases; además de administrar la institución; es una persona líder y aprende a desarrollar su práctica pedagógica en un ambiente multigrado caracterizado por la diversidad y la heterogeneidad de la población estudiantil, aspectos visibilizados en el material audiovisual que deben observar las personas estudiantes.

De acuerdo con los argumentos que anteceden, la experiencia didáctica es promotora del pensamiento crítico en el estudiantado que se encuentra en formación, con una alta expectativa del análisis y las reflexiones generadas que posibilitan nuevas formas de ver el mundo, así como valorar el papel del profesorado unidocente y los escenarios multigrado, espacios donde es factible el desarrollo de habilidades para la vida.

El objetivo de este trabajo es compartir los hallazgos de la valoración realizada por el estudiantado participante en la aplicación de la estrategia Cine-foro virtual, la cual tuvo como propósito la reflexión sobre el papel de la persona unidocente y en general, la importancia de las escuelas unidocentes en el contexto rural, por medio de un ejercicio que facilita la comprensión de la relación entre la teoría y la práctica.

\section{DESARROLLO DE LA EXPERIENCIA}

Esta experiencia se lleva a cabo de forma virtual asincrónica en el curso Educación Rural y Escuela Unidocente que imparte la Escuela de Formación Docente de la UCR, Sede Rodrigo Facio, durante el I Ciclo lectivo de 2020. El trabajo se inscribe en el enfoque de investigación cualitativa al pretender la comprensión de la perspectiva del estudiantado (Hernández, Fernández y Baptista, 2010), en este caso se profundiza sobre experiencias y opiniones en relación con la participación en el Cine-foro virtual.

La población participante se conformó por 27 estudiantes y correspondió al total del estudiantado de formación docente que comparte un perfil académico similar; pues se halla matriculado en el noveno ciclo del Plan de Estudio de la Carrera de Bachillerato y Licenciatura en Educación Primaria; por lo tanto, la muestra se considera intencional y homogénea (Hernández, Fernández y Baptista, 2010).

La sistematización de esta experiencia, realizada en tres fases que se describen más adelante, se considera retrospectiva, ya que se lleva a cabo una vez concluida su aplicación. La metodología se enfoca en la ejecución del Cine-foro virtual como una estrategia didáctica de análisis y reflexión sobre el objeto de estudio, con un énfasis en la relación teoría-práctica.

\section{Instrumento de recolección de datos}

El instrumento para la recolección de la información consistió en un cuestionario autoadministrado dirigido a la población estudiantil, su objetivo fue evaluar la experiencia didáctica. Se construyó por medio de la aplicación Google Forms, fue validado por una persona docente del Departamento de Docencia Universitaria de la UCR e incluyó ocho ítems de respuesta cerrada y tres de respuesta abierta. 
Los ítems de la sección objetiva se elaboraron para recolectar información sobre: a) el momento antes de participar en las actividades: espacio de consulta sobre la claridad y objetividad de las instrucciones de participación y el desarrollo de la estrategia; el beneficio de las temáticas y contenidos abordados en el curso; y el contar con los conocimientos previos para la participación; b) el momento posterior a la participación en las actividades: se consultó a la población estudiantil acerca del contenido desarrollado con la aplicación de la estrategia; el reconocimiento del valor de la escuela unidocente, el proceso de reflexión personal en torno a la función docente y las características de las escuelas rurales unidocentes; y el aporte de la lectura para la construcción de aprendizajes significativos; y c) la calificación asignada a la experiencia didáctica: se solicitó al estudiantado asignar una calificación a la experiencia, de acuerdo con la calidad y objetividad de esta, la escala utilizada fue de 1 a 10, donde 1 era la calificación más baja, y 10 , la más alta.

Por su parte, los ítems de la sección subjetiva pretendieron recolectar información sobre los aprendizajes obtenidos por la población estudiantil, las recomendaciones o las sugerencias para la mejora de la experiencia didáctica y los argumentos dirigidos a la posible replicación de dicha experiencia en el futuro para el curso Educación Rural y Escuela Unidocente.

El análisis de la información recolectada se realiza de manera cualitativa y se organiza en las siguientes categorías:

a) Diseño de la experiencia didáctica Cine-foro virtual: corresponde a la etapa de planificación y se define como el conjunto de actividades diseñadas para que el estudiantado, a partir del documental y su participación en las actividades del Cine-foro virtual, logren inferir la importancia de la escuela unidocente en el contexto rural de Costa Rica, así como el papel de la persona docente. Se pretende que el diálogo que se suscite entre las personas participantes favorezca esa inferencia. .

b) Ejecución de la experiencia didáctica: incumbe a la etapa de implementación de las actividades del Cine-foro virtual y se refiere a la pertinencia de estas para el logro de los objetivos propuestos desde el programa del curso Educación Rural y Escuela Unidocente, ante la sustitución de la gira educativa.

c) Aprendizajes logrados por la población estudiantil: es el proceso formativo de evaluación donde el estudiantado describió los aprendizajes logrados por medio de su participación en las actividades de la experiencia. Según los resultados se organiza la información en: caracterización de la escuela unidocente, caracterización del maestro y la maestra unidocente, el contexto rural y la estrategia didáctica.

d) Valoración de la experiencia didáctica por parte del estudiantado: se enmarca en la necesidad de conocer la opinión de la población estudiantil respecto a las recomendaciones o sugerencias de mejora en el diseño didáctico y su aplicación, así como la replicación de las actividades en el futuro para el curso Educación Rural y Escuela Unidocente. La información se describe en dos líneas: recomendaciones al diseño didáctico y replicación de la experiencia en el futuro.

Finalmente, la información se discute y contrasta a la luz de los argumentos teóricos y, de esta forma, se obtienen la síntesis y las reflexiones finales.

\section{Rol de las personas docente y estudiante en el desarrollo de la experiencia}

El rol de la persona docente es activo, cumple la función de facilitadora y guía de los procesos de aprendizaje; construye la estrategia de forma clara y concreta, con anticipación epistemológica de los fundamentos teóricos que guían el quehacer pedagógico, debe considerar los hechos educativos como parte 
de la problematización de la situación actual, así como reconocer al sujeto que participa como agente de cambio social (Ramírez, 2008). Asimismo, fomenta en el estudiantado una participación autónoma (en lo individual) y cooperativa (en lo grupal), promueve una verdadera interacción en la modalidad virtual (Arango, 2003), sin olvidar que su accionar coadyuve en la comprensión de las realidades educativas sociales.

El rol de la persona estudiante también es activo, es sujeto protagonista de su aprendizaje, demuestra habilidades de participación, análisis, reflexión y comunicación; al mismo tiempo, asume una posición crítica ante la situación planteada en la experiencia. Logra diferenciar la realidad existente en la que está inscrito y la realidad estudiada con la cual se puede confrontar (Ramírez, 2008); es decir, reconstruye y cuestiona otras opciones sobre el objeto de estudio.

\section{Fases para el desarrollo de la experiencia didáctica}

En una fase previa, se evidenció la necesidad de sustituir la estrategia de inmersión estudiantil en la realidad del contexto unidocente, por el Cine-foro virtual acompañado de un avance en el portafolio de evidencias digital que el estudiantado realizaba desde el inicio del curso. Las instrucciones de la estrategia se alojaron en un espacio de trabajo semanal en el entorno virtual del curso, ubicado en la Plataforma Mediación Virtual UCR, un sitio de trabajo en línea que permite la interacción entre la persona docente y el estudiantado. La secuencia de actividades, flexible y aplicable a diferentes contextos, contó con la ventaja del acompañamiento de los siguientes recursos digitales:

1. Guía de avance en el portafolio digital de evidencias, compuesta por dos secciones:

En la primera sección se describe el documental por observar: Ser y tener (2002), Francia, el cual está disponible en el enlace: https://www.youtube.com/watch?v=U8AKTL5LP6I.

Descripción: con una duración es de 104 minutos, el documental destaca principios básicos de la escuela multigrado que se comparten con las escuelas unidocentes de Costa Rica.

En este sentido, el recurso recoge la cotidianidad de una escuela unidocente en un espacio rural, desde el primero hasta el último día de clases, permite visualizar cómo un docente logra desarrollar el proceso de aprendizaje con un grupo heterogéneo de estudiantes de preescolar y primaria. Así se logra apreciar las estrategias metodológicas aplicadas para atender, de manera simultánea, diversos niveles, la educación personalizada, los recursos didácticos, los agrupamientos flexibles, el uso del entorno para enriquecer el currículo, la participación de las familias y la comunidad en la educación de sus hijos e hijas. De manera muy clara se percibe cómo el docente interactúa afectivamente con el estudiantado, para atender las diversas situaciones que se presentan dentro y fuera del aula; además que permite identificar cómo el grupo de estudiantes se ayudan entre sí en la dinámica de aula.

Posterior a su observación el estudiantado contesta por escrito y de forma cooperativa, las siguientes actividades:

- Se enfoca la atención sobre uno de los siguientes temas y se desarrolla: características del docente, características del estudiantado, relación docente-estudiante, relación estudiante-estudiante, proceso de aprendizaje o relación de la escuela con su entorno.

- Se responden las cuestionantes: a) Mientras veían el documental, ¿qué preocupaciones les surgieron?; b) ¿Qué sentimientos provocó el documental en ustedes? ¿Por qué?, y c) Si pudieran cambiar algo dentro de la realidad que se presenta en el documental, ¿qué cambiarían y cómo lo harían? 
En la segunda sección se solicitó al estudiantado realizar la lectura obligatoria "Capítulo Il, Perfil del educador o de la educadora en la escuela Unidocente, unitaria o multigrado, su formación y capacitación", de Rocío Alvarado Cruz y Arnobio Maya Betancour (2009). Luego de eso, observar el vídeo corto llamado Una niña, una escuela, el cual está disponible en el enlace: https://www.youtube.com/ watch?v=luCK5JrOMCl.

Con una duración de catorce minutos, ubicado geográficamente en Cuba, el vídeo presenta la situación de una niña que asiste a una escuela unidocente y muestra otra realidad educativa en este tipo de escuela, la enseñanza unidocente unigrado e individualizada. El traslado de la maestra y la niña a la escuela demuestra las dificultades de acceso a algunas de estas instituciones; además que las condiciones de infraestructura y los recursos disponibles para la enseñanza y el aprendizaje no siempre son lo mejor. De esta forma, se muestra una realidad similar a la que puede vivir el estudiantado y la persona docente de una escuela unidocente costarricense, así se incrementa el valor de su presencia en las comunidades rurales.

Luego de observar el vídeo corto, el estudiantado debió mencionar, de forma escrita, al menos tres principios que fundamentan el trabajo de la persona docente unidocente, además de comentar algunas de sus características, funciones y roles, retos y obstáculos.

2. Guía de tarea-individual: documento que describe la forma de participación del estudiantado en el Cine-foro virtual, esto incluye las preguntas generadoras para la discusión: ¿por qué el documental se llama Ser y tener? Y, ¿qué nuevas resignificaciones surgen sobre la escuela unidocente a partir de lo observado?

En la tabla 1 se presenta el cronograma de desarrollo de las actividades de esta fase.

TABLA 1

Cronograma de actividades para el desarrollo de la experiencia

\begin{tabular}{cl}
\multicolumn{1}{c}{ Fecha } & \multicolumn{1}{c}{ Actividades por realizar } \\
7 al 14 de mayo & $\begin{array}{l}\text { Ejecución de las actividades de la guía de trabajo en el portafolio digital - Trabajo cooperativo, lo } \\
\text { cual involucra la observación del documental Sery tener - Individual. }\end{array}$ \\
7 al 11 de mayo & $\begin{array}{l}\text { Ejecución de las actividades propuestas en la guía de tarea - Individual: primer aporte al foro. } \\
11 \text { al } 14 \text { de mayo }\end{array}$ \\
& $\begin{array}{l}\text { Ejecución de las actividades de la guía de tarea - Individual: participación con un mínimo de dos } \\
\text { réplicas a los comentarios y aportes de los demás integrantes. }\end{array}$ \\
\hline
\end{tabular}

Fuente: elaboración propia (2020).

Se aclara que las guías incorporaron rúbricas y escalas de evaluación con sus respectivos indicadores, fueron calificadas y consideradas como parte de la evaluación sumativa del curso. La evaluación formativa se llevó a cabo por medio de la retroalimentación docente sobre la calificación de los productos, durante el desarrollo del foro se realizó mediante la formulación de comentarios y preguntas generadoras dirigidas al estudiantado.

En la siguiente fase de desarrollo, el estudiantado procedió a la ejecución de las actividades:

a) Ejecución de las actividades de la guía de avance en el portafolio digital de evidencias, la cual se desarrolló de forma grupal y asincrónica, mediante los dos procesos ya citados: observación del documental Ser y Tener y contestación de ejercicios; así como la realización de la lectura teórica, acompañada de un ejercicio de comprensión. Se observó el vídeo corto Una niña, una escuela y con base en los insumos de estos recursos el estudiantado procedió a la resolución de las actividades de la guía de trabajo del portafolio.

b) Ejecución individual-asincrónica de la tarea de participación en el Cine-foro virtual, acompañado de la retroalimentación y la síntesis de los contenidos relacionados con el documental. El 
tiempo de participación para subir las respuestas de las preguntas del ejercicio 1, fue a partir del 7 de mayo (7:00 p.m.) y se extendió hasta el 11 de mayo (12:00 m.d.). Las intervenciones para aportar o consultar al resto de participantes, se podían realizar desde el 8 hasta el 14 de mayo (5:00 p.m.).

c) Evaluación de la participación en las actividades, a través de la revisión del portafolio digital de evidencias y las intervenciones en el Cine-foro virtual. Ambos trabajos fueron presentados por medio del entorno virtual en la Plataforma Mediación Virtual UCR.

La última fase de valoración consistió en la evaluación de la experiencia por medio de la aplicación del cuestionario. En esta fase, de los 27 estudiantes que conformaron la población participante durante el desarrollo de la experiencia, 24 completaron con éxito la totalidad del formulario, 18 mujeres y seis hombres. Esta evaluación aporta al proceso de análisis y discusión que se realiza sobre la experiencia didáctica que se comparte.

\section{RESULTADOS}

Se organizan los resultados y la discusión según las categorías de análisis declaradas previamente, a la luz de las valoraciones del estudiantado y la persona investigadora.

En relación con el "Diseño de la experiencia didáctica Cine-foro virtual", se establece una clara evidencia de que para la población estudiantil las instrucciones de participación y el desarrollo de la experiencia fueron claras y objetivas (92\%); además de que las temáticas abordadas en el curso aportaron en su desempeño a lo largo de las actividades realizadas (92\%). Otro aspecto relevante es que casi la totalidad del estudiantado (96\%), indicó que contó con los conocimientos previos para participar en las actividades. Se afirma, por lo tanto, que estos conocimientos que forman parte de la estructura cognitiva previa en el estudiantado, relacionados con los temas abordados en el curso antes de participar en la experiencia didáctica: caracterización de las zonas rurales de Costa Rica y educación rural, se conectan con la nueva información a modo de anclaje (Ausubel, 1983): la escuela unidocente y el papel de la persona docente, generando aprendizajes significativos.

Por otro lado, se subraya que, para el estudiantado, la lectura y las preguntas generadoras cumplieron un papel primordial en el cumplimiento de las actividades (96\%), esto fue posible por la intención y el diseño lógico de las actividades de aprendizaje y la evaluación propuestas; pues estas componen efectivamente una secuencia didáctica que, con la adecuada mediación docente, se dirige al logro de metas.

Al respecto de las secuencias didácticas, según Tobón, Pimienta y García (2010), estas buscan "el logro de determinadas metas educativas, considerando una serie de recursos" (p. 20). Por ello es por lo que, en este caso, se promovió el uso de varios recursos didácticos. En síntesis, se afirma que el conjunto de actividades diseñadas en la experiencia fue efectivo para favorecer el acercamiento al contexto rural y reflexionar sobre el papel de la persona unidocente, a pesar de que se sustituyó la gira educativa por la participación virtual asincrónica en las actividades del Cine-foro virtual.

Sobre la "Ejecución de la experiencia didáctica", para el estudiantado, posibilitó la caracterización de la persona unidocente, se logró identificar sus funciones y roles, y el reconocimiento de los retos y los obstáculos que se pueden presentar en su labor (92\%), aspectos que se evidenciaron en las participaciones registradas en el foro que se habilitó en el entorno virtual del curso.

A partir del ejemplo patente en el documental Ser y tener, la mayoría de la población estudiantil aportó ideas sobre el papel de la persona unidocente, se destacó una reivindicación de la labor docente, al reconocer la diversidad de funciones pedagógicas y administrativas que atiende. 
Autores como Morera, Angulo y Torres (2010), concuerdan con esa necesidad de reivindicar la labor pedagógica y la gestión institucional, y recalcan

[...] la necesidad de proveer a los centros educativos rurales de materiales y recursos de apoyo a los procesos de enseñanza y aprendizaje, y la necesidad de formar profesionales como una visión de trabajo donde se articule los objetivos del curriculum con las necesidades e intereses de los estudiantes, de la institución y la comunidad (p. 138).

Dichos aspectos se reflejaron no solo en la práctica pedagógica observada en el material audiovisual propuesto, sino también en el proceso de reflexión causado con la participación en el Cine-foro virtual.

Se reitera que, a pesar de que no fue posible realizar la gira educativa presencial, la población estudiantil, en su totalidad, está de acuerdo con que, por medio de la participación en las actividades de la experiencia virtual propuesta, se logró identificar el valor de la escuela unidocente (100\%). Es así como se manifiesta la funcionalidad del documental y las actividades propuestas, de manera que se comprende el alto valor pedagógico que representan las escuelas unidocentes (Alvarado y Maya, 2009), al tiempo que se da pie para el estudio de sus potencialidades, así como las dificultades que enfrentan en la actualidad.

Otro elemento por destacar en la ejecución de la experiencia es que prácticamente todo el grupo de estudiantes (96\%), señaló estar de acuerdo en que esta permitió reflexionar sobre la función docente y las características de las escuelas rurales unidocentes; solo un sujeto participante indicó estar en desacuerdo con tal afirmación. Se establece una clara evidencia de que las actividades, en particular, posibilitaron la identificación de las diferencias entre la práctica pedagógica rural unidocente y la urbana, esta última, en general, es en la que la población estudiantil ha estado inmersa desde edades muy tempranas.

Sobre el reto de caracterizar las escuelas unidocentes, se destaca que, en la discusión generada en el Cine-foro virtual, brotaron aspectos como la ubicación diversa en zonas rurales dispersas rodeadas de un entorno natural particular, una comunidad social y educativa claramente definida y la atención por una persona docente. Respecto a las personas docentes, Alvarado y Maya (2009), confirman que "tienen que desempeñar una serie de funciones en las que debe atender: el desarrollo curricular, la gestión escolar y el trabajo con la comunidad educativa" (p. 14). A ello se suma un ambiente de aprendizaje, generalmente, multigrado.

Por consiguiente, el estudiantado, en su mayoría (79\%), exteriorizó que la lectura utilizada aportó en la construcción de aprendizajes relacionados con la escuela unidocente y el perfil de la persona docente; sin embargo, se reflexiona en la posibilidad de revisar el material para identificar si es posible sustituirlo por uno mejor o acompañarlo con algún otro recurso que profundice sobre la realidad educativa unidocente en el contexto rural costarricense. Por ejemplo, entre las conclusiones de la investigación "Las escuelas unidocentes de Costa Rica: fortalezas y limitaciones", Chaves y García (2013), concluyen que "Los datos dan cuenta de carencias en cuanto a infraestructura, material didáctico, equipo tecnológico, presupuesto, sobrecarga de trabajo para el personal docente, falta de oportunidades para el desarrollo profesional, falta de asignaturas especiales, entre otros." (p. 23). Carencias que poco contribuyen al fomento de una educación pública de calidad y son considerados como aspectos críticos que también deben ser analizados por la población estudiantil.

Ahora bien, respecto a los "Aprendizajes logrados por la población estudiantil", en la tabla 2 se sintetizan los resultados para cada una de las subcategorías emergentes. 
TABLA 2

Aprendizajes logrados por la población estudiantil

\section{Caracterización de la escuela unidocente}

- Mejor compren- . sión de su dinámica y metodología . de trabajo.

- Característicasque posee, así como su organización.

- Relación de los conceptos escuela unidocente y escuela multigrado.

- Importancia de estas instituciones y la integración del aprendizaje.

- La existencia de escuelas unidocentes o multigrado en otros países.

\section{Caracterización del maestro y la maestra unidocente}

\section{Contexto rural}

Comprensión del rol - Importancia de que la . unidocente.

\section{Características y funcio-} nes, las cuales sobrepasan los elementos meramente pedagógicos.

- Importancia de su presencia en la comunidad y los desafíos a los cuales se enfrenta.

- Metodología de trabajo unidocente, esto incluye las estrategias, el trato personalizado y la interacción entre estudiantes en un ambiente multigrado.

Estilo de vida que lleva el maestro o la maestra unidocente.

- Una persona estudiante, en particular, hace mención de que "aprendió sobre el rol de la persona estudiante". persona docente contextualice los aprendizajes.

- Hacer uso de los recursos del entorno.

- En particular, una persona estudiante sugirió que el significado de Ser y tener, título del documental, le insinuó la importancia de que esta frase caracterice a las escuelas rurales, como, por ejemplo, en el compartir tiempos, tareas y la convivencia y el crecimiento que ese compartir aporta.

- Importancia del trabajo en conjunto con las familias, así como lo enriquecedor de la interacción entre los niños y las niñas de diferentes edades. emociones, además de

\section{Estrategia didáctica}

El documental permitió salirse de la rutina y motivó a la participación en el foro.

Importancia de la crítica para responder a las cuestionantes que dirigían la experiencia.

- Se promovió la expresión de opiniones y percepciones de los integrantes del grupo, así como el análisis de posteriores interpretaciones y aportes.

- La estrategia requiere una adecuada planeación por etapas y refuerza los temas y contenidos del curso.

Dos personas estudiantes, en particular, señalaron que:

1. "el cine-foro y el portafolio me parecen provechosas en cuanto nos permiten ordenar la información obtenida por medio de los vídeos (película y documental) y evidenciar los aprendizajes significativos por medio de la reflexión".

2. "el portafolio virtual permite construir el aprendizaje adquirido mediante la realización de las actividades. Y el cine-foro permitió dar un punto de vista crítico de la película" (Estudiantes del curso Educación Rural y Escuela Unidocente, 2020).

Fuente: elaboración propia (2020).

De acuerdo con los hallazgos, se infiere que los aprendizajes del estudiantado están ligados a la intención de la experiencia y, por lo tanto, se demuestra de forma contundente que esta fortalece la caracterización del papel de la persona unidocente y la importancia de las escuelas unidocentes en el contexto rural. Por medio del diálogo participativo sobre las bondades y las dificultades de la enseñanza multigrado y la práctica pedagógica unidocente, es posible evidenciar en las respuestas del estudiantado el contraste entre teoría y práctica, se observa el desarrollo de aprendizajes que se vinculan con algunos de los supuestos teóricos de la pedagogía crítica, tales como: la humanización y la contextualización educativas, así como la transformación de la realidad social (Ramírez, 2008).

En general, la experiencia aporta al aprendizaje de metodologías y el uso de recursos que coadyuvan en el trabajo docente futuro, así motiva al desarrollo del pensamiento crítico, con una mirada emancipadora de las realidades sociales y educativas que se erigen en el contexto rural.

Se concluye que los aprendizajes del estudiantado reiteran que el documental es acertado para el logro de los aprendizajes esperados, además de que no hubiera sido posible explicitar el contenido del curso a través del uso único o exclusivo de la teoría. Empero de no contar con la inmersión en un escenario rural unidocente, el grupo de estudiantes demostró que la experiencia permite, desde la modalidad virtual asincrónica, analizar y reflexionar, con criticidad, el valor de la escuela rural unidocente, así como las funciones, roles, retos y obstáculos que enfrentan sus actores (aspectos desarrollados en el trabajo escrito que acompañó al foro). 
Finalmente, acerca de la "Valoración de la experiencia didáctica por parte del estudiantado", en la tabla 3 se resumen las recomendaciones al diseño didáctico y los argumentos brindados para su posible replicación en el futuro.

TABLA 3

Resultados sobre la valoración de la experiencia didáctica por parte del estudiantado

\begin{tabular}{ll}
$\begin{array}{l}\text { Recomendaciones generales } \\
\text { al diseño didáctico }\end{array}$ & \multicolumn{1}{c}{$\begin{array}{c}\text { Argumentos para replicar la experiencia en el futuro } \\
\text { - } \begin{array}{l}\text { Extender el tiempo destinado a la parti- } \\
\text { cipación en el cine-foro virtual. }\end{array}\end{array}$} \\
$\begin{array}{l}\text { Acompañar la estrategia con espacios de } \\
\text { trabajo virtual sincrónico. }\end{array}$ & $\begin{array}{l}\text { Sobre el documental se menciona que es atinente para el análisis de la es- } \\
\text { cuela rural unidocente y el ambiente multigrado, la información y perspec- } \\
\text { tiva mostrada es valiosa para quienes no conocen una escuela unidocente. }\end{array}$ \\
- $\begin{array}{l}\text { Presentar un único listado de pasos o } \\
\text { instrucciones de las actividades por } \\
\text { realizar }\end{array}$ & $\begin{array}{l}\text { Sobre la experiencia: es de fácil ejecución, atractiva y motivante; se facilita } \\
\text { la comprensión de las funciones de la persona unidocente; se vincula la } \\
\text { teoría y la práctica, en relación con la labor docente; permite conocer me- }\end{array}$ \\
$\begin{array}{l}\text { Apuntar más a la discusión en el foro, } \\
\text { ejercitar la contrastación de ideas sobre } \\
\text { la variación de conocimientos posterior } \\
\text { a la participación y crear pequeños gru- } \\
\text { pos de discusión por temas, así como la } \\
\text { utilización de diferentes vídeos. }\end{array}$ & $\begin{array}{l}\text { miento crítico; además de la interacción con el resto de los y las integrantes } \\
\text { del grupo; relaciona los sentimientos personales con la función docente } \\
\text { reflejada en el documental, aunado a la identificación de los roles, retos y } \\
\text { desafíos. }\end{array}$ \\
\hline
\end{tabular}

Fuente: elaboración propia (2020).

A pesar de que la pandemia provocada por el virus responsable de la COVID-19 ocasionó la adaptación educativa para cumplir con los aprendizajes esperados en el curso, la experiencia del Cine-foro virtual fue valorada positivamente por el estudiantado participante; pues le asignó una calificación promedio de 9 . Además, el $100 \%$ de dicha población recomendó que las actividades se apliquen bajo la misma modalidad para el curso Educación Rural y Escuela Unidocente en el futuro.

Conforme a lo anterior, se identifica el valor de la experiencia didáctica debido no solo a la excelente calificación otorgada por el estudiantado, sino porque las recomendaciones atañen aspectos plausibles de mejora para futuras experiencias. Las opiniones del grupo de estudiantes ofrecen una perspectiva clara de que la planificación de la experiencia y los recursos utilizados fueron adecuados.

Desde el enfoque socio-crítico se promueve que el estudiantado en formación docente sea un intérprete crítico de las situaciones didácticas y pedagógicas en las cuales participa; por lo tanto, la experiencia involucró el trabajo cooperativo y el alcance de aprendizajes con una dimensión individual, ética y social (Oxfam, 2009, citado en Sección de Educación Primaria, 2014). Asimismo, se valora que la guía y el acompañamiento de la persona docente encargada del diseño y la ejecución de la experiencia; por lo tanto, resulta fundamental para acarrear un proceso organizado, ordenado y contextualizado, de manera que se ofrezca al estudiantado la oportunidad de lograr, en el medio virtual, aprendizajes significativos.

\section{SÍNTESIS Y REFLEXIONES FINALES}

Sobre el diseño de la experiencia, se concluye que el Cine-foro virtual es una estrategia efectiva que promueve la comunicación asertiva entre el estudiantado y, entre este y la persona docente; por otro lado, el dinamismo y el trabajo asincrónico que acompañaron su aplicación robustecen el desempeño estudiantil, lo cual promueve el diálogo en torno a las diversas percepciones sobre las temáticas que se abordaron, en este caso, mediante el análisis y la reflexión sobre un ejemplo de la realidad rural unidocente expresada en el documental. 
Sobre la ejecución, la población estudiantil expresó su acuerdo con la propuesta, el 100\% de ella participó en las actividades y el $89 \%$, la evaluó. El tipo de recursos utilizados facilitó el desarrollo de las actividades formuladas; sin embargo, se valora la recomendación de elaborar una sola guía de actividades por ejecutar, de manera que el estudiantado se apegue a la realización de un solo cuerpo de procedimientos.

Pese a la dificultad de no poder llevar a cabo la gira educativa de inmersión en el contexto rural unidocente, la experiencia y los aprendizajes logrados por el grupo de estudiantes representó una forma de innovar en la práctica docente universitaria, lo cual fue posible gracias a la interacción estudiantil y docente, además del uso de recursos digitales y tecnológicos, elementos que permearon el accionar educativo en el contexto de la pandemia ocasionada por el virus responsable de la COVID-19.

La implementación de la secuencia de actividades fue coherente con el alcance de los objetivos del curso y permitió, por medio del diálogo social participativo llevado a cabo en la modalidad virtual, la construcción de una comunidad de aprendizaje por medio de un profundo proceso de análisis y cuestionamiento, en el cual se compartieron conocimientos, experiencias e inquietudes respecto a la realidad educativa rural unidocente. Como resultado, la experiencia se puede adaptar a diversos cursos y contextos de aprendizaje en el área educativa; además, podría trasladarse al entorno universitario presencial una vez realizada su contextualización a las circunstancias y el momento histórico-social que se vive.

En contraste, se destaca que las actividades facilitaron que el estudiantado se vinculara emocionalmente con la función de la persona unidocente, a la vez que valoró los elementos del entorno como parte del desarrollo de la práctica pedagógica en los contextos rurales, los cuales están caracterizados por la heterogeneidad.

El cumplimiento de la intención para la cual fue diseñada y ejecutada la experiencia revela que, en la modalidad virtual, también existe la posibilidad de generar un acercamiento a la realidad del ambiente de enseñanza unidocente y multigrado, por medio de la construcción de conocimientos, de acuerdo con las líneas de interacción y relación entre la teoría y la práctica, así como la acción reflexión. Es por lo anterior que la experiencia podría ser replicada en el futuro, así lo aseguró el 100\% del estudiantado que participó en el proceso de evaluación.

Por otra parte, frente a las recomendaciones brindadas por el estudiantado, no se puede evadir la importancia del acompañamiento docente de forma virtual sincrónica, de manera que se susciten espacios para la reflexión que encausen las opiniones y las participaciones de la población estudiantil hacia los objetivos de aprendizaje. Finalmente, se recomienda para la replicación de esta experiencia en el futuro, contar con vídeos de prácticas pedagógicas en escenarios multigrado, propias del contexto educativo costarricense, las cuales permitan al estudiantado aprender y valorar los diversos contextos rurales unidocentes. En ese mismo sentido, se llama la atención sobre la necesidad de que se investigue sobre los diversos escenarios rurales unidocentes de Costa Rica y reflejar su realidad en recursos audiovisuales que faciliten, al personal docente universitario y al estudiantado, su conocimiento, mediante la modalidad virtual. 


\section{REFERENCIAS}

Alvarado, R. y Maya, A. (2009). La escuela unidocente: un reto y una oportunidad para la educación en la comunidad rural. Recuperado de https://ceccsica.info/sites/default/files/content/Volumen_48.pdf

Arango, M. (2003). Foros virtuales como estrategia de aprendizaje. Debates Latinoamericanos, 2. Recuperado de https://revistas.rlcu.org.ar/index.php/Debates/article/download/33/19/

Ausubel, D. (1983). Teoría del aprendizaje significativo. Fascículos de CEIF, 1:1-10:

Barberà, E. (2005). La evaluación de competencias complejas: la práctica del portafolio. Educere, 9 (31):497-504. Recuperado de https://www.redalyc.org/pdf/356/35603110.pdf

Bustos, A. (2011). La escuela rural. Granada, España: Octaedro.

Cartín, D. (2018). Escuelas Públicas Unidocentes en Costa Rica: Historia y Situación Actual (1960-2016). Recuperado de https://www.mep.go.cr/indicadores_edu/BOLETINES/uni.pdf

Chaves, L. y García, J. (2013). Las escuelas unidocentes en Costa Rica : fortalezas y limitaciones. Revista Educación, 37(1):1-27. Disponible en https://revistas.ucr.ac.cr/index.php/educacion/article/ view/10626/10297

DEDUN (2020). Las estrategias didácticas. Universidad de Costa Rica. Recurso digital del curso de Didáctica Universitaria.

Hernández, R., Fernández, C y Baptista, M. (2010). Metodología de la investigación. D.F, México: McGraw-Hill.

Morera, D. Angulo, L. y Torres, N. (2010). El proceso pedagógico de la escuela rural unidocente y multigrado centroamericana. Curridabat, Costa Rica: Uruk Editores.

Piedra, L. (2010). La transición de un modelo individualista e internista a uno más social y colaborativo en la formación universitaria. Revista De Ciencias Económicas, 28 (2):313-322. Recuperado de https://revistas.ucr.ac.cr/index.php/economicas/article/view/7091

Programa Estado de la Nación. (2017). Sexto informe estado de la educación. (1 ed.) Recuperado de https://www.estadonacion.or.cr/educacion2017/assets/ee6-informe-completo.pdf

Ramírez, R. (2008). La pedagogía crítica. Una manera ética de generar procesos educativos. Revista Folios, 28:108-119. Recuperado de https://www.redalyc.org/pdf/3459/345941358009.pdf

Sección de Educación Primaria. (2014). Reestructuración Integral del Plan de Estudio de la Carrera de Bachillerato y Licenciatura en Educación Primaria. Escrito sin publicar.

Tobón, S.; Pimienta, J. y García, J. (2010). Secuencias Didácticas: aprendizaje y evaluación de competencias. Recuperado de http://files.ctezona141.webnode.mx/200000004-8ed038fca3/secuenciasdidacticastobon-120521222400-phpapp02.pdf 